\title{
Programa de autogerenciamento da dor crônica no idoso: estudo piloto*
}

\section{Self-management of chronic pain in the elderly: pilot study}

Fania Cristina Santos ${ }^{1}$, Polianna Mara Rodrigues de Souza², Silvana Angélica Coelho Nogueira ${ }^{3}$, Isabel Clasen Lorenzet ${ }^{3}$, Bianca Figueiredo Barros ${ }^{3}$, Luciana Paula Dardin ${ }^{4}$

* Recebido da Disciplina de Geriatria e Gerontologia da Universidade Federal de São Paulo (UNIFESP), Disciplina de Geriatria e Gerontologia. São Paulo, SP.

\section{RESUMO}

JUSTIFICATIVA E OBJETIVOS: O programa de autogerenciamento da dor (AGD) inclui educação sobre dor, treinamento para identificação e modificação de pensamentos negativos, estabelecimento de metas, exercícios de relaxamento e terapias físicas com o objetivo de reduzir a dor, melhorar o humor e o funcionamento psicossocial. O objetivo foi avaliar o desenho metodológico e sua viabilidade em estudar a eficácia do AGD no tratamento da dor crônica em idosos e, secundariamente, avaliar sua eficácia comparando-o a um método educativo controle.

MÉTODO: Foram avaliados os aspectos sociodemográficos, aspectos sobre a dor crônica e sua mensuração, impacto em funcionalidade, qualidade de vida, cognição e emoção utilizando-se: avaliação sociodemográfica, escala analógica visual (EAV) de dor, “Ge-

1. Médica Geriatra, Coordenadora do Serviço de Doenças Osteoarticulares e Dor da Disciplina de Geriatria e Gerontologia (DIGG) da Universidade Federal de São Paulo. São Paulo, SP, Brasil.

2. Médica Geriatra, Assistente do Serviço de Dor e doenças Osteoarticulares da Disciplina de Geriatria e Gerontologia (DIGG) da Universidade Federal de São Paulo, São Paulo, SP, Brasil.

3. Médica do Serviço de Dor e doenças Osteoarticulares da Disciplina de Geriatria e Gerontologia (DIGG) da Universidade Federal de São Paulo. São Paulo, SP, Brasil.

4. Fisioterapeuta do Serviço de Dor e doenças Osteoarticulares da Disciplina de Geriatria e Gerontologia (DIGG) da Universidade Federal de São Paulo. São Paulo, SP, Brasil.

Endereço para correspondência:

Fania Cristina dos Santos

Disciplina de Geriatria e Gerontologia / DIGG - UNIFESP

Rua Francisco de Castro $\mathrm{n}^{\circ} 105$ - Vila Clementino

04020-050 São Paulo/SP, Brasil

Fone/Fax: (11) 5575-4848

E-mail: faniacs@uol.com.br riatric pain measurement" (GPM), escala de Katz e de Lawton (avaliação de funcionalidade), mini-exame do estado mental (MEEM), Geriatric Depressive Scale (GDS) e SF-36.

RESULTADOS: Foram incluídos 20 indivíduos (12 no G1/intervenção e 8 no G2/controle), todos do gênero feminino, com idade média 73,7 no G1 e 78,2 no G2. A intensidade média de dor ao início do estudo foi $18,6 \mathrm{~mm}$ no G1, e $16,6 \mathrm{~mm}$ no G2, quando utilizada a EAV e pontuação média de 21,8 no G1 e 19,7 no G2, utilizando-se o GPM. Ao final do estudo a intensidade média de dor segundo a EAV foi $16,7 \mathrm{~mm}$ no $\mathrm{G1}(\mathrm{p}=0,342)$ e 41,2 $\mathrm{mm}$ no $\mathrm{G} 2(\mathrm{p}=0,006)$. A pontuação com o GPM foi 19,7 no $\mathrm{G} 1(\mathrm{p}=0,400)$ e 25,8 no $\mathrm{G} 2(\mathrm{p}=0,346)$. As pontuações pela escala Katz foi 5,8 no início e 5,9 no final do estudo no G1 com 5,4 no início e 5,6 no final no G2 (p= $0,198)$. Pela escala de Lawton houve melhora funcional estaticamente significante no G1 $(p=0,040)$, o mesmo não ocorrendo no $\mathrm{G} 2(\mathrm{p}=0,148)$ e a diferença entre os grupos foi significante $(\mathrm{p}=0,032)$. Os valores médios obtidos com os tratamentos propostos pela SF-36 não diferiram significativamente entre os grupos.

CONCLUSÃO: A intervenção de AGD melhorou a independência funcional do idoso, para as atividades instrumentais de vida diária, e demonstrou tendência em melhorar a intensidade da dor e qualidade de vida.

Descritores: Dor, Idoso, Gerenciamento, Tratamento.

\section{SUMMARY}

BACKGROUND AND OBJECTIVES: The pain self-management program (PSM) includes education about pain, training to identify and change negative thinking, establishment of goals, relaxation exercises and physical therapies aiming at decreasing pain and improving mood and psychosocial functioning. This 
study aimed at evaluating the methodological performance and the feasibility of studying PSM effectiveness to treat chronic pain in the elderly and, secondarily, at evaluating its effectiveness as compared to a control educational method.

METHOD: Social-demographic aspects, chronic pain and its evaluation, impact on functionality, quality of life, cognition and emotion were evaluated using: socio-demographic evaluation, pain visual analog scale (VAS), Geriatric pain measurement (GPM), Katz and Lawton scales (functionality evaluation), mental state mini exam (MSME), Geriatric Depressive Scale (GDS) and SF-36.

RESULTS: Participated in this study 20 females (12 in the G1/intervention and 8 in G2/control groups), mean age of 73.7 in G1 and of 78.2 in G2. Mean pain intensity in the beginning of the study was $18.6 \mathrm{~mm}$ in G1 and $16.6 \mathrm{~mm}$ in $\mathrm{G} 2$, when VAS was used, and 21.8 in G1 and 19.7 in G2 with GPM. At the end of the study, mean pain intensity according to VAS was $16.7 \mathrm{~mm}$ for G1 (p $=-.342)$ and 41.2 for $\mathrm{G} 2(\mathrm{p}=0.006)$. GPM scores were 19.7 for $\mathrm{G} 1(\mathrm{p}=0.400)$ and 25.8 for $\mathrm{G} 2(\mathrm{p}=0.346)$. Katz scale scores were 5.8 in the beginning and 5.9 at the end of the study for G1 and 5.4 in the beginning and 5.6 at the end for $\mathrm{G} 2(\mathrm{p}=0.198)$. Lawton scale showed statistically significant functional improvement for G1 $(p=0.040)$, the same not being true for G2 $(p=0,148)$ with significant difference between groups $(p=0.032)$. Mean values obtained with SF-36 were not statistically significant between groups.

CONCLUSION: PSM intervention has improved functional independence of the elderly for daily life activities and has shown a trend to decrease intensity and improve quality of pain.

Keywords: Elderly, Management, Pain, Treatment.

\section{INTRODUÇÃO}

O envelhecimento populacional é um fenômeno mundial. Segundo a Organização das Nações Unidas (ONU) a parcela da população com mais de 60 anos é a que mais cresce. Em 2000 havia cerca de 400 milhões de idosos no mundo. Estimativas apontam que em 2050 serão mais de um bilhão e meio, o que representará cerca de $20 \%$ da população mundial ${ }^{1,2}$.

No Brasil não é diferente. Na década de 1960 havia no país cerca de três milhões de pessoas com 60 anos ou mais, em 2000 essa parcela da população já somava mais de 14 milhões de pessoas, ou seja, quase 9\% da população brasileira. Estimativas do Instituto Brasileiro de
Geografia e Estatística (IBGE) apontam que em 2020 os idosos representarão $15 \%$ da população brasileira, saltando em 2050 para 18\%; o que corresponderá a cerca de 38 milhões de pessoas. O Brasil será o $6^{\circ}$ país com maior número de idosos.

À medida que a população envelhece maior é a prevalência de problemas crônicos de saúde e incapacidades funcionais associadas. Dentre esses problemas encontram-se diversas doenças associadas à dor crônica.

Estima-se que $20 \%$ a $50 \%$ dos idosos provenientes da comunidade apresentam problemas relacionados à presença de dor, esse número aumenta para $45 \%$ a $80 \%$ em pacientes institucionalizados, podendo ser ainda maior nos internados e, em muitos desses casos, a dor não é adequadamente reconhecida e tratada. Estudos mostram que mais de $50 \%$ dos idosos portadores de dor crônica não recebem o seu controle adequado e mais de $25 \%$ morrem sem obter o seu controle ${ }^{3-5}$. Em idosos com deficiências cognitivas, o diagnóstico e tratamento da dor podem tornar-se um problema ainda maior, o que, em parte, se justifica pela maior dificuldade em sua avaliação ${ }^{5-8}$. Por isso a tendência atual em considerar a dor como o quinto sinal vital, devendo, pois, ser abordada em toda avaliação clínica.

As principais causas de dor no idoso, especialmente dor crônica, são doenças osteoarticulares (principalmente as degenerativas), osteoporose e suas consequências, fraturas, doença vascular periférica, neuropatia diabética e outras neuropatias periféricas, neuralgia pós herpética, síndrome dolorosa pós-acidente vascular encefálico (AVE), dor do membro fantasma, polimialgia reumática, lombalgias, doenças neoplásicas, desordens musculoesqueléticas e quaisquer condições com prejuízo de mobilidade ${ }^{3-7}$.

Entre os idosos, a dor crônica é a mais comum, gerando inúmeras consequências graves e potencialmente debilitantes dentre as quais se podem citar depressão, ansiedade, isolamento social, distúrbio do sono, dificuldades de movimentação e deambulação, prejuízo da autoavaliação de saúde e aumento da necessidade de gastos com cuidados de saúde ${ }^{3,5,6,8}$. Acredita-se que o estresse gerado pela dor crônica atue como um fator contribuinte para lentificação de processos de reabilitação e de recuperação de lesões, redução de capacidade funcional e aumento de dependência nos $\operatorname{idosos}^{6,8}$. As lesões podem ser ainda maiores na presença de comorbidades e polifarmácia ${ }^{2,6}$, fatores que muitas vezes limitam o uso de tratamentos farmacológicos otimizados. 
Intervenções que incorporam componentes educativos, cognitivos e comportamentais tem se mostrado eficazes na melhora da dor e da incapacitação física e psicossocial. Assim, abordagens não farmacológicas da dor crônica vêm sendo desenvolvidas com o objetivo de aumentar a habilidade dos pacientes em lidar com suas dores ${ }^{9,10}$.

O programa de autogerenciamento da dor (AGD) é uma dessas abordagens e inclui educação sobre a dor, treinamento para identificação e modificação de pensamentos negativos, estabelecimento de metas, exercícios de relaxamento e o uso de terapias físicas com o objetivo de reduzir a dor, melhorar o humor e o funcionamento psicossocial ${ }^{12-14}$.

O objetivo primário deste estudo piloto foi avaliar a viabilidade do desenho metodológico escolhido e possibilitar a melhor estruturação de estudos de maiores proporções para posteriormente estudar a eficácia do programa de AGD em idosos, ou seja, observar se a técnica de AGD, comparada a um programa educacional passivo, é efetiva em reduzir a dor, e melhorar a funcionalidade e a qualidade de vida de idosos com dor persistente.

O objetivo secundário foi avaliar a eficácia do método AGD nesta pequena população do estudo.

\section{MÉTODO}

Após aprovação pelo Comitê de Ética em Pesquisa da Universidade Federal de São Paulo (UNIFESP), parecer $\mathrm{n}^{\mathrm{o}} 0023 / 08$, realizou-se este estudo prospectivo, controlado e aleatório. Os participantes foram recrutados nos ambulatórios da Disciplina de Geriatria e Gerontologia (DIGG) da UNIFESP, sendo todos provenientes da comunidade. Os critérios de inclusão do estudo foram: idade de 60 anos ou mais, portadores de dor crônica com duração maior ou igual há 3 meses, capacidade e motivação para participar do estudo e assinatura do termo de consentimento livre e esclarecido. Critérios de exclusão: câncer atual, cirurgia nos últimos 6 meses ou cirurgia prevista para os 6 meses seguintes.

Foram avaliados aspectos sociodemográficos; características da dor crônica; impacto em funcionalidade, qualidade de vida, cognição e emoção. Foram utilizados os seguintes instrumentos: inventário de dados sociodemográficos, escala analógica visual (EAV) de dor para avaliação unidimensional da dor (intensidade), Geriatric pain measurement (GPM) para avaliação multidimensional da dor (pontuação máxima de 42 = pior avaliação da dor), escala de Katz e de Lawton para a avaliação de funcionalidade, miniexame do estado mental (MEEM) para avaliação cognitiva, Geriatric Depressive Scale (GDS) para avaliação de alterações emocionais e SF-36 para mensuração da qualidade de vida. Realizada aleatorização para dois grupos: Grupo 1 (G1) intervenção e Grupo 2 (G2) controle. Os dois grupos receberam cartilhas educativas (técnica educacional passiva) com orientações sobre dor, mas apenas o G1 participava de sessões semanais do AGD por sete semanas seguidas, conforme explanado na tabela 1. As cartilhas educativas abordavam os temas principais do programa AGD: princípios básicos da dor; o exercício e a atividade física no gerenciamento da dor; atividades prazerosas e significativas para melhorar a dor; desafiando pensamentos negativos e lidando com picos de dor e contratempos; terapia não medicamentosa e medicamentosa para a dor; e consideradas o nosso método educativo controle.

Todos receberam ligações telefônicas semanais para acompanhamento e, ao final de setes semanas, todos foram submetidos a reavaliações, sendo utilizados os mesmos instrumentos iniciais.

A análise estatística foi realizada com teste de ANOVA com medidas repetidas paramétricas e não paramétricas com significância de $\mathrm{p}<0,05$.

\section{RESULTADOS}

A amostra foi composta por 20 indivíduos (12 no G1 e 8 no G2), todos do gênero feminino, e idades médias de 73,7 e 78,2 anos, G1 e G2, respectivamente. Ainda, caracterizando a amostra, segundo a avaliação unidimensional da dor, utilizando a EAV, as intensidades médias de dor foram de 18,6 mm no G1, e 16,6 mm no G2, e segundo a abordagem multidimensional da dor, utilizando-se o GPM, as pontuações médias foram de 21,8 no G1 e 19,7 no G2. Observou-se tratar de grupos homogêneos, pois as diferenças entre os grupos não foram estatisticamente significantes.

Os resultados obtidos ao final do estudo, ou seja, aqueles obtidos com os programas propostos, quanto à avaliação unidimensional da dor, as intensidades médias de dor segundo a EAV, mostram que no G1 houve uma redução da dor para $16,7 \mathrm{~mm}$, ou seja, pequena melhora na dor de $1,9 \mathrm{~mm}$; contudo, não estatisticamente significante $(\mathrm{p}=$ 0,342). No G2, houve aumento da dor para $41,2 \mathrm{~mm}$, ou seja, houve uma piora da dor de 24,6 mm; piora essa significante, do ponto de vista estatístico $(\mathrm{p}=0,006)$. Mas, comparando-se os dois grupos entre si, não se observou diferença significante na intensidade da dor $(\mathrm{p}=0,144)$. Quanto ao desfecho avaliação multidimensional da dor, 
Tabela 1 - Intervenção no grupo de autogerenciamento da dor

Números de Sessões: Tópicos

Sessão 1: Introdução aos

princípios básicos da dor

Sessão 2: O papel dos exercícios físicos: atividade física no gerenciamento da dor

Sessão 3: Envolvendo-se com atividades prazerosas e significativas

Sessão 4: Desafiando pensamentos negativos. Lidando com picos de dor e os contratempos

Sessão 5: Terapias analgésicas sem medicamentos: calor e frio. Lidando com picos de dor e seus contratempos

Sessão 6: Medicamentos e noções sobre outras terapias complementares no tratamento da dor crônica

Sessão 7: Plano de gerenciamento da dor. Encerramento
Conteúdo e Atividades Principais

Revisar definições, tipos e mecanismos de dor crônica. Discutir mitos sobre a dor em idosos. Enfatizar os objetivos do gerenciamento dor crônica. Discutir sinais e sintomas que exigem atenção médica. Explicar com detalhes o programa AGD. Apresentar técnicas de solução de problemas para o gerenciamento da dor.

Discutir o papel do exercício no gerenciamento da dor: problemática da perda de condicionamento, tipos de exercícios, dicas para começar um programa de exercícios. Demonstração e prática de exercícios específicos. Apresentar técnicas de relaxamento e respiração como estratégias eficazes no gerenciamento da dor. Praticar relaxamento muscular progressivo e respiração abdominal.

Discutir sobre como a dor crônica pode limitar a participação em atividades significativas e prazerosas. Usar a solução de problemas para desenvolver planos individuais para aumentar essas atividades. Discutir estratégias para atividades de caminhadas e fundamentos para evitar a inatividade. Praticar relaxamento.

Discutir o papel do pensamento e avaliação sobre a dor para determinar respostas emocionais e comportamentais à dor. Ajudar os participantes a identificarem pensamentos negativos que possam ter em resposta à dor. Praticar e desafiar os pensamentos positivos que são maneiras eficazes para se lidar com a dor. Discutir estratégias para lidar com picos de dor e contratempos. Praticar relaxamento.

Descrever fundamentos para o uso de terapias sem medicamentos. Descrever e praticar a aplicação de compressas quentes e frias. Revisar precauções ao usar compressas quentes e frias. Continuar a discussão sobre lidar com picos de dor e contratempo no gerenciamento da dor. Praticar relaxamento.

Descrever o papel dos medicamentos no gerenciamento da dor. Discutir os principais tipos de medicamentos para dor. Descrever o uso de terapias complementares no gerenciamento da dor (outras terapias como distração, hipnose, acupuntura...). Discutir passos para se tomar decisão sobre todas as terapias de dor.

Discutir a manutenção dos ganhos obtidos através do programa. Revisão de como lidar com picos de dor e contratempos no gerenciamento da dor. Revisar os planos escritos de manutenção individual para cada participante. os resultados mostram: redução na pontuação do GPM de 21,8 para 19,7 no $\mathrm{G} 1$, contudo, redução não significante $(\mathrm{p}=0,400)$, e aumento de 22,3 para 25,8 no $\mathrm{G} 2(\mathrm{p}=$ $0,346)$, novamente não estatisticamente significante $(\mathrm{p}=$ 0,346). Também não foi observada diferença significativa, do ponto de vista estatístico, entre os grupos $(\mathrm{p}=0,401)$. Referente à funcionalidade, as pontuações na escala de Katz para o nível de independência nas atividades básicas de vida diária, foram no G1 de 5,8 no início e de 5,9 no final do estudo. No G2, a pontuação foi de 5,4 no início e de 5,6 no final, sem diferença significativa entre os grupos $(\mathrm{p}=0,198)$.

Também, referente à funcionalidade, mas relativo ao nível de independência nas atividades instrumentais de vida diária, observaram-se com a escala de Lawton, melhora funcional estaticamente significante no G1 (p = $0,040)$, mas o mesmo não ocorreu no $\mathrm{G} 2(\mathrm{p}=0,148)$. E comparando-se os grupos, a diferença na funcionalidade foi significante $(\mathrm{p}=0,032)$.

Para as avaliações de transtornos do humor utilizando-se o GDS, as pontuações obtidas foram: G1 de 3,85 no início e 3,10 no final, e no G2 de 4,14 inicial e 7,2 no final, sem diferenças significativas entre os grupos ( $p=$ 0,198). O mesmo ocorreu para as avaliações cognitivas, ou seja, segundo o MEEM as médias obtidas foram de 26,6 inicial e final, e de 24,6 inicial e final, G1 e G2, respectivamente, sem diferenças significativas entre os grupos $(\mathrm{p}=0,107)$. 
Quanto às dimensões da qualidade de vida, os valores médios obtidos com os tratamentos propostos não diferiram significativamente, do ponto de vista estatístico. Referentes à dimensão vitalidade, observaram-se as médias de 61,15 inicial e 61,5 final no G1, e 66,4 inicial e 65 final no $\mathrm{G} 2(\mathrm{p}=0,985)$. Referente ao estado geral, no G1 os valores médios foram de 66,6 inicial e 76,1 final, e no G2 de 58,1 inicial e 68,8 final $(\mathrm{p}=0,294)$. Na dimensão emocional, os valores observados foram no G1 de 74,2 inicial e 66,6 final; e no G2 de 47,5 inicial e 79,9 final (p=0,771).

\section{DISCUSSÃO}

A dor crônica é bastante prevalente no idoso e com o acelerado envelhecimento da população, tornar-se-a cada vez mais comum na prática diária do profissional de saúde. Em decorrência das alterações na metabolização de fármacos e maior possibilidade de eventos adversos e iatrogenia nessa população, torna-se fundamental a adoção de medidas não farmacológicas associadas ao tratamento farmacológico da dor, possibilitando, em muitas situações, uso de menor número de drogas e menores doses, com redução dos riscos de efeitos indesejáveis, mantendo um controle adequado da dor.

Estratégias de AGD, que incorporam várias técnicas para o tratamento da dor, são consideradas eficazes em diminuir a dor crônica e melhorar a função e humor em jovens. Pouco se sabe sobre a eficácia dessa terapia em idosos. Pretendeu-se aqui, além de apresentar um modelo de tratamento coadjuvante no manuseio da dor crônica da população idosa, verificar a eficácia do mesmo para os indivíduos idosos.

Neste estudo, uma eficácia superior do programa AGD nos idosos com dor crônica (em comparação com o programa educativo controle) no controle da dor, melhora funcional; tanto física quanto psicossocial, e melhora na qualidade de vida não foi comprovada, contudo, várias limitações foram encontradas. O "Power" do estudo (probabilidade de detectar uma diferença), que varia com a concordância dos indivíduos no âmbito local e o tamanho do efeito, não foi suficiente para se detectar diferenças. A amostra foi pequena, pois se tratou de um estudo piloto, e com muitas variáveis a considerar.

Uma das primeiras dificuldades foi observada logo no início do estudo. Mesmo com aulas e roteiro previamente estruturados para a realização das sessões de AGD, existiram dificuldades no "funcionamento dinâmico" no G1, pois em muitos aspectos, o perfil psicossocial e nível educacional dos integrantes interferem na dinâmica global do trabalho. Outra questão que necessita revisão para melhor estruturação num estudo maior, é o conteúdo do livreto educativo, considerado aqui como método educativo passivo e controle. Observou-se que existiram muitas dúvidas dos participantes em relação ao entendimento do mesmo, e talvez houvesse a necessidade de uma linguagem ainda mais simplificada para os idosos. Quanto à caracterização da amostra, observou-se que a mesma foi composta por 20 idosas caucasianas, com intensidades médias de dor crônica de $18,6 \mathrm{~mm}$ no G1 e $16,6 \mathrm{~mm}$ no G2. Nesta avaliação unidimensional da dor, ou seja, avaliação apenas da intensidade dolorosa, verificou-se tratar de dor crônica de leve intensidade. Quanto à sua avaliação multidimensional, as pontuações médias de dor, segundo o GPM, foram de 21,8 e de 22,3, G1 e G2, respectivamente, denotando tratar-se de dor crônica com poucas/moderadas dimensões comprometidas.

Referindo-se aos desfechos no estudo, e segundo as alterações nas intensidades da dor para os tratamentos propostos, não se observaram diferenças estatisticamente significantes entre os programas $(p=0,144)$. Contudo, houve uma tendência à diminuição da dor com o programa AGD, e piora da mesma com o programa controle, sendo que em relação a essa piora, a diferença foi estatisticamente significante. Isto poderia sugerir uma tendência à melhora da dor com o programa AGD, e talvez, uma superioridade na eficácia fosse demonstrada numa amostra adequada.

Quanto aos aspectos multidimensionais da dor no idoso utilizando-se o instrumento GPM, ou seja, aspectos referentes à intensidade e qualidade da dor, não ocorreu diferença estatisticamente significante entre os programas. Aqui, também se observou uma tendência à diminuição da dor com o programa AGD, e piora da mesma com o programa apenas educativo, pois as diferenças aqui encontradas não foram estatisticamente significantes $(\mathrm{p}=0,401)$.

Segundo a avaliação de humor com os tratamentos propostos, também se observou que, ao final do estudo, o grupo controle apresentou maior pontuação no GDS, ou seja, pior avaliação de humor para o grupo controle, contudo, piora não significante, do ponto de vista estatístico $(p=0,198)$. Novamente se verificou aqui, uma tendência a melhor eficácia do programa AGD em melhorar o humor, pois no grupo controle a pontuação no GDS tendeu a piorar. $\mathrm{O}$ mesmo foi observado para a dimensão emocional da qualidade de vida, onde os participantes do programa AGD tenderam a melhora. Houve o benefício da convivência em grupo, e das atividades interativas oferecidas no programa, e isto pode ter influenciado a socialização, e os aspectos emocionais dos participantes. Segundo as avaliações cognitivas, não foram observados 
diferenças significativas entre os grupos $(p=0,107)$. E o mesmo ocorreu quanto à qualidade de vida, em todas as dimensões avaliadas: vitalidade, estado geral, e aspectos emocionais, contudo, como já mencionado, observou-se uma tendência a maior eficácia do programa AGD na dimensão emocional.

Referente à independência funcional para as atividades de vida de diária (AVD) não se observou diferença estatisticamente significativa entre os grupos. E quanto ao nível funcional para as atividades instrumentais de vida diária (AIVD), aqui se demonstrou melhor eficácia para o programa AGD $(p=0,032)$, apesar da melhora funcional ter sido pequena. Mais uma vez, reflete-se aqui, maior eficácia da intervenção $\mathrm{AGD}$, pois funcionalidade para indivíduos idosos é de muita importância.

Assim, verificaram-se nesta casuística que os resultados obtidos não forneceram boas evidências da eficácia da intervenção de AGD para idosos com dor crônica, comparando-se com o método educativo controle. Apenas observou-se uma melhora na independência funcional do idoso, segundo as AIVD, e também, certa tendência em melhorar a intensidade dolorosa, compreendendo a multidimensionalidade da dor, ou melhor, intensidade e qualidade da dor. Tais dados sugerem que, talvez, numa maior amostragem, a eficácia de um programa multimodal de AGD da dor crônica no idoso possa ser verificada. A eficácia dessa terapia na dor crônica em jovens já foi demonstrada. Mas, para um estudo com maior casuística envolvendo idosos haveria a necessidade de vários ajustes metodológicos, e principalmente um maior "power". Apresentando em detalhes um programa de AGD da dor crônica no idoso, como aqui se verificou, nota-se que ele é muito simples, consta de sessões semanais, duração de duas horas cada, num total de 7 sessões, portanto, não sendo necessários grandes gastos, ponto muito importante no nosso meio.

Para melhor avaliação com o tratamento proposto seria necessário outro estudo com ajustes metodológico, e este projeto-piloto integra estudo maior da Disciplina de Geriatria e Gerontologia da UNIFESP.

\section{CONCLUSÃO}

A intervenção de AGD melhorou a independência funcional do idoso, para as atividades instrumentais de vida diária, e demonstrou tendência em melhorar a intensidade dolorosa, compreendendo a multidimensionalidade da dor, ou melhor, intensidade e qualidade da dor.

\section{REFERÊNCIAS}

1. Gambaro RC, Santos FC, The KB, et al. Avaliação de dor no idoso: proposta de adaptação do Geriatric Pain Measure para a língua portuguesa. Rev Bras Med 2009;66(3):62-5.

2. McCleane G. Pharmacological pain management in the elderly patient. Clin Interv Aging 2007;2(4):637-43.

3. Chopra A. Pain management in the older patient. Clin Geriat 2006;14(3):40-6.

4. AGS panel on persistent pain in older persons. The management of persistent pain in older persons. J Am Geriatr Soc 2002;50(6 Suppl):S205-S24.

5. AGS panel on persistent pain in older persons. Pharmacological management of persistent pain in older persons. J Am Geriatr Soc 2009;57:1331-46.

6. Karp JF, Shega JW, Morone NE, et al. Advances in understanding the mechanisms and management of persistent pain in older adults. Br J Anaesth 2008;101(1):111-20.

7. Fine PG. Chronic pain management in older adults: special considerations. J Pain Symptom Manage 2009;38(2 Suppl):S4-S14.

8. Kaasalainen S, Molloy DW. Pain and aging. Geriatrics today. J Can Geriatr Soc 2001;32-7.

9. Ersek M, Turner JA, McCurry SM, et al. Efficacy of a self-management group intervention for elderly persons with chronic pain. Clin J Pain 2003;19(3):156-67.

10. American Geriatric Society. The management of persistent pain in older persons. J Am Geriatr Soc 2002;50;S205-24.

11. McCracken LM, Turk DC. Behavioral and cognitive-behavioral treatment for chronic pain: outcome, predictors of outcome, and treatment process. Spine 2002;27(22):2564-73.

12. Rossy LA, Buckelew SP, Dorr N, et al. A meta-analysis of fibromyalgia treatment interventions. Ann Behav Med 1999;21(2):180-91.

13. Turk DC. Cognitive-behavioral approach to the treatment of chronic pain patients. Reg Anesth Pain Med 2003;28(6):573-9.

14. Devine EC. Meta-analysis of the effect of psychoeducational interventions on pain in adults with cancer. Oncol Nurs Forum 2003;30(1):75-89.

Apresentado em 03 de março de 2011.

Aceito para publicação em 02 de setembro de 2011. 